\title{
Vaccination of Preterm Infants
}

\section{Bernhard Resch*}

Research Unit for Neonatal Infectious Diseases and Epidemiology, Division of Neonatology, Department of Paediatrics, Medical University of Graz, Austria

Immunization of premature infants is usually recommended to occur at the chronological age comparable with term infants without correcting for gestational age following national infant vaccination programs. The main reason behind this recommendation is that preterm and low birth weight infants are at increased risk of infections in general and suffer from an increased frequency and severity of vaccine preventable infections. The need for timely vaccination of preterm infants is therefore great. However, vaccination is more likely to be delayed in preterm than in term infants $[1,2]$

\section{Immune Response of Preterm Infants}

At 8 weeks of age preterm infants have lower absolute counts of lymphocytes, T cells, B cells, and T helper cells and a lower CD4/CD8 ratio than term infants. By the age of 7 months $B$ cell numbers in the preterm group have reached term equivalent, but the reduced absolute lymphocyte count, total $\mathrm{T}$ cell count, and $\mathrm{T}$ helper count persist [3]. During the third trimester of pregnancy an intense development of the $\mathrm{B}$ cell receptor repertoire actually occurs, thus, limiting the range of antigens recognised by preterm B cells compared to term infants. However, there is evidence that premature exposure to antigens can accelerate this development. This means that extremely low gestational age newborns may actually have a more diverse antibody repertoire by the time they reach the corrected age of 40 weeks than infants who are born at term [4], and this is an important argument against delayed immunisation of preterm infants.

The impaired functioning of immune defences to infection in especially preterm infants and their implication for vaccination have recently been reviewed in detail [5]. Shortly, external barrier functions potentially impact on immunization through the immunoregulatory functions of antimicrobial peptides and the impaired functions of the innate immune system through dendritic cells and macrophage dysfunction. The vaccine induced systemic protection involves B cells, cytotoxic T cells or T helper cells, or combined responses reflecting the interplay between the humoral and cell mediated immunity.

\section{Effectiveness of Immunization in Preterm Infants}

Vaccine responsiveness can be determined by either vaccine immunogenicity or efficacy studies. The latter is based on large numbers of individuals to demonstrate how much of a reduction in disease incidence occurs in vaccinated compared to unvaccinated persons. And this is the main problem for preterm infants: low frequency of diseases associated with low numbers of infants that would be required for such clinical trials. A systematic review was published recently determining vaccine responsiveness to tetanus, diphtheria, meningococcal $\mathrm{C}$ and pneumococcal conjugates, Haemophilus influenzae type B, polio and pertussis [6]. The focus of this review was to determine vaccine immunogenicity using immunological correlates of protection and to estimate whether an individual preterm infant was immune based on a "putative protective antibody level". Data showed that immunogenicity in premature infants was vaccine specific and whilst highly protective for the toxoid and inactivated preparations, responses to the subunit preparations were less optimal.

A physiological reason why premature infants may respond even better to vaccination than anticipated is the kinetics of trans-placental transfer of maternal antibody. Maternofetal transport of immune globulin G starts around the $17^{\text {th }}$ gestational week, is at equilibrium around the $33^{\text {rd }}$ week, and reaches up to twofold higher values in the neonate at term. Thus, preterm infants, depending on their gestation, will have low or absent concentrations of maternal antibody. While this in part explains susceptibility to infections, it may provide an advantage in response to vaccines [1].

\section{Adverse Events}

A small number of infants may have apnoea with or without bradycardia temporally associated with immunization. Rates have been reported to range between 13 and 25\% [7-9]. Resurgence or an increase of bradycardia and desaturation episodes or isolated desaturation episodes have been reported in $43 \%$ of infants immunized with a hexavalent vaccine [10]. Apneas, bradycardias and desaturations have been observed to occur more often in preterm infants with a chronological age below 67 to 70 days at the time of first immunization $[11,12]$. The risk factors for, and recurrence rate of apnoea with subsequent immunisations are unknown, which makes planning for subsequent immunizations for these highly vulnerable infants difficult. In a retrospective surveillance cohort analysis including all preterm infants with a cardiorespiratory event following first immunization $18 \%$ (95\% confidence interval 6-31\%) had recurrent apnoea with subsequent immunization [13]. Possible risk factors for recurrence included lower birth weight and ongoing hospitalization for complications relating to prematurity. No preterm infant with recurrent apnoea had a third episode of apnoea with subsequent immunization. For infants in the NICU without apnea during the 24 hours immediately before immunization, younger age, smaller size, and more severe illness at birth were reported to be important predictors of post-immunization apnea besides pre-immunization apnea being the most important one [12]. Thus, the American Academy of Pediatrics recommended immunization of preterm and low birth weight infants when they are medically stable [14], and the Committee for Infectious Diseases and Vaccination of the German Academy of Pediatrics and Adolescent Medicine [15] recommended very immature preterm infants $(<28$ weeks of gestational age) to be immunized during their first hospitalization irrespective of a history of cardiorespiratory events.

\section{Conclusion}

While absolute primary antibody responses might be lower

*Corresponding author: Bernhard Resch, Research Unit for Neonatal Infectious Diseases and Epidemiology, Division of Neonatology, Department of Paediatrics Medical University of Graz, Auenbruggerplatz 34/2, 8036 Graz, Austria, Tel: +43 316385 81134; Fax: +43 318385 12678; E-mail: Bernhard.resch@medunigraz.at

Received January 15, 2014; Accepted January 17, 2014; Published January 19, 2014

Citation: Resch B (2014) Vaccination of Preterm Infants. J Neonatal Biol 3: e107. doi:10.4172/2167-0897.1000e107

Copyright: (c) 2014 Resch B. This is an open-access article distributed under the terms of the Creative Commons Attribution License, which permits unrestricted use, distribution, and reproduction in any medium, provided the original author and source are credited. 
in preterm infants immunized according to chronological age compared with term infants, the majority of preterm infants achieve concentrations generally accepted to be protective. In clinical practise the first vaccination (Diphtheria-tetanus-pertussis-polio-Haemophilus influenzae type B-hepatitis B and pneumococcal and meningococcal C conjugates) might be given following one to three days without a history of apneas/bradycardias during the first hospitalization with subsequent observation for cardiorespiratory events preferably when the preterm infant reached 67 to 70 days of life. In case of post-immunization cardiorespiratory events the subsequent vaccination should be administered under close monitoring during rehospitalisation for 48 to 72 hours.

\section{References}

1. Bonhoeffer J, Siegrist CA, Heath PT (2006) Immunisation of premature infants. Arch Dis Child 91: 929-935.

2. Esposito S, Serra D, Gualtieri L, Cesati L, Principi N (2009) Vaccines and preterm neonates: why, when, and with what. Early Hum Dev 85: S43-45.

3. Berrington JE, Barge D, Fenton AC, Cant AJ, Spickett GP (2005) Lymphocyte subsets in term and significantly preterm UK infants in the first year of life analysed by single platform flow cytometry. Clin Exp Immunol 140: 289-292.

4. Bauer K, Zemlin M, Hummel M, Pfeiffer S, Karstaedt J, et al. (2002) Diversification of Ig heavy chain genes in human preterm neonates prematurely exposed to environmental antigens. J Immunol 169: 1349-1356.

5. Baxter D (2010) Impaired functioning of immune defenses to infection in premature and term infants and their implications for vaccination. Hum Vaccin 6: 494-505.

6. Baxter D (2010) Vaccine responsiveness in premature infants. Hum Vaccin 6 : 506-511.
7. Schulzke S, Heininger U, Lücking-Famira M, Fahnenstich H (2005) Apnoea and bradycardia in preterm infants following immunisation with pentavalent or hexavalent vaccines. Eur J Pediatr 164: 432-435.

8. Ellison VJ, Davis PG, Doyle LW (2005) Adverse reactions to immunization with newer vaccines in the very preterm infant. J Paediatr Child Health 41: 441-443.

9. Flatz-Jequier A, Posfay-Barbe KM, Pfister RE, Siegrist CA (2008) Recurrence of cardiorespiratory events following repeat DTaP-based combined immunization in very low birth weight premature infants. J Pediatr 153: 429-431.

10. Omeñaca F, Garcia-Sicilia J, García-Corbeira P, Boceta R, Romero A, et al. (2005) Response of preterm newborns to immunization with a hexavalent diphtheria-tetanus-acellular pertussis-hepatitis B virus-inactivated polio and Haemophilus influenzae type b vaccine: first experiences and solutions to a serious and sensitive issue. Pediatrics 116: 1292 - 1298.

11. Sen S, Cloete $Y$, Hassan K, Buss $P$ (2001) Adverse events following vaccination in premature infants. Acta Paediatr 90: 916-920.

12. Klein NP, Massolo ML, Greene J, Dekker CL, Black S, et al. (2008) Risk factors for developing apnea after immunization in the neonatal intensive care unit Pediatrics 121: 463-469.

13. Clifford V, Crawford NW, Royle J, Lazzaro T, Danchin M, et al. (2011) Recurrent apnoea post immunisation: Informing re-immunisation policy. Vaccine 29: 5681-5687.

14. Saari TN; American Academy of Pediatrics Committee on Infectious Diseases (2003) Immunization of preterm and low birth weight infants. American Academy of Pediatrics Committee on Infectious Diseases. Pediatrics 112: 193-198.

15. Committee for Infectious Diseases and Vaccination of the German Academy of Pediatrics and Adolescent Medicine (2009) Respiratory monitoring in former premature infants $(<28 \mathrm{GW})$ following primary immunization. Comment. Monatsschr Kinderheilkd 157: 173-176. 